\title{
Observations of Bright Massive Stars Using Small Size Telescopes
}

\author{
Sopia Beradze and Nino Kochiashvili \\ E. Kharadze Abastumani Astrophysical Observatory, Ilia State University \\ email: sopia.beradze.1@iliauni.edu.ge
}

\begin{abstract}
The size of a telescope determines goals and objects of observations. During the latest decades it becomes more and more difficult to get photometric data of bright stars because most of telescopes of small sizes do not operate already. But there are rather interesting questions connected to the properties and evolution ties between different types of massive stars. Multiwavelength photometric data are needed for solution of some of them. We are presenting our observational plans of bright Massive X-ray binaries, WR and LBV stars using a small size telescope.

All these stars, which are presented in the poster are observational targets of Sopia Beradze's future $\mathrm{PhD}$ thesis. We already have got very interesting results on the reddening and possible future eruption of the massive hypergiant star P Cygni. Therefore, we decided to choose some additional interesting massive stars of different type for future observations. All Massive stars play an important role in the chemical evolution of galaxies because of they have very high mass loss - up to $10^{-4} \mathrm{M}_{\odot} /$ a year. Our targets are on different evolutionary stages and three of them are the members of massive binaries. We plan to do UBVRI photometric observations of these stars using the $48 \mathrm{~cm}$ Cassegrain telescope of the Abastumani Astrophisical Observatory.
\end{abstract}

Keywords. X-ray Binaries, WR Stars, LBV, Multi-Wavelength Photometry, Small Size Telescopes

\section{Acknowledgemets}

The authors are grateful to E.Kharadze Abastumani Astrophysical Observatory, Ilia State University, Georgia, for funding our travel to New Zealand. N.K. thanks conference organizers for covering part of her local expences at the conference. 